\title{
Respectful modelling: Addressing uncertainty in dynamic system models for molecular biology
}

DOI:

10.1016/j.tibtech.2016.12.008

\section{Document Version}

Accepted author manuscript

Link to publication record in Manchester Research Explorer

\section{Citation for published version (APA):}

Tsigkinopoulou, A., Murtuza Baker, S., \& Breitling, R. (2017). Respectful modelling: Addressing uncertainty in dynamic system models for molecular biology. Trends in Biotechnology, 35(6), 518-529.

https://doi.org/10.1016/j.tibtech.2016.12.008

\section{Published in:}

Trends in Biotechnology

\section{Citing this paper}

Please note that where the full-text provided on Manchester Research Explorer is the Author Accepted Manuscript or Proof version this may differ from the final Published version. If citing, it is advised that you check and use the publisher's definitive version.

\section{General rights}

Copyright and moral rights for the publications made accessible in the Research Explorer are retained by the authors and/or other copyright owners and it is a condition of accessing publications that users recognise and abide by the legal requirements associated with these rights.

\section{Takedown policy}

If you believe that this document breaches copyright please refer to the University of Manchester's Takedown Procedures [http://man.ac.uk/04Y6Bo] or contact uml.scholarlycommunications@manchester.ac.uk providing relevant details, so we can investigate your claim.

\section{OPEN ACCESS}




\title{
Respectful modelling: Addressing uncertainty in dynamic system models for molecular biology
}

Areti Tsigkinopoulou, Syed Murtuza Baker, Rainer Breitling

Address

Manchester Centre for Synthetic Biology of Fine and Speciality Chemicals (SYNBIOCHEM), Manchester Institute of Biotechnology, Faculty of Science and Engineering, University of Manchester, 131 Princess Street, Manchester, M1 7DN, United Kingdom.

Corresponding author:

Full name and email: Rainer Breitling (rainer.breitling@manchester.ac.uk)

Postal address: Manchester Institute of Biotechnology, University of Manchester, 131

Princess Street, Manchester, M1 7DN, United Kingdom

Telephone: +441613065117

\section{Keywords}

Ensemble modelling, uncertainty, distributions, biological systems, Bayesian statistics

\begin{abstract}
Although there is still some scepticism within the biological community regarding the value and significance of quantitative computational modelling, important steps are continually being taken in order to enhance its accessibility and predictive power. We view these developments as essential components of an emerging "respectful modelling” framework, which has two critical aims: 1) Respecting the models themselves and facilitating the reproduction and update of modelling results by other scientists. 2) Respecting the predictions of the models, and rigorously quantifying the confidence associated with the modelling results. This respectful attitude will guide the design of higher-quality models and facilitate the use of models in modern applications, such as engineering and manipulating microbial metabolism by synthetic biology.
\end{abstract}

\section{Computational models in current research: Success and scepticism}

Quantitative computational models of cellular pathways and circuits are essential tools for generating clear, testable predictions about the behaviour of complex cellular machineries [1, 2]. Their use is currently moving beyond the proof-of-concept stage towards real-world applications, such as engineering and optimising biological microorganisms to produce specific chemicals and biofuels [3-5], as has been shown most successfully for various 
terpenoids [6] and for succinic acid [7]. Their applications in identifying potential drug targets in metabolic or signalling pathways are also rapidly advancing [8-10]. An increasing number of success stories demonstrate that computational models have a lot to offer to biologists, from surveying cellular development [11] and exploring signalling pathways [12] and genetic circuits [13], to investigating potential treatments for cancer [14, 15]. However, modelling is not yet part of the mainstream of biological practice, even in fields like synthetic biology that intend to embrace an engineering approach to manipulating biological complexity [16-18].

Major progress is also being made in the case of genome-scale models [19-21], which match enzyme-coding genes with predicted reactions in metabolic pathways. Until recently, their nature limited the application of genome-scale models to stoichiometric constraint-based approaches, studying the fluxes through the system without being able to predict metabolite levels or the dynamics of responses to internal or external perturbations. However, efforts are being made to incorporate detailed information on enzyme regulation and kinetic mechanisms into such constraint-based models, thus transforming them into genome-scale kinetic models of metabolism [22-24].

We aim to outline (Fig. 1) "respectful modelling," an emerging set of closely related concepts and techniques that together enhance and advance earlier modelling approaches to make computational models both more approachable and more relevant for the work of experimental biologists.

\section{Respectful Modelling}

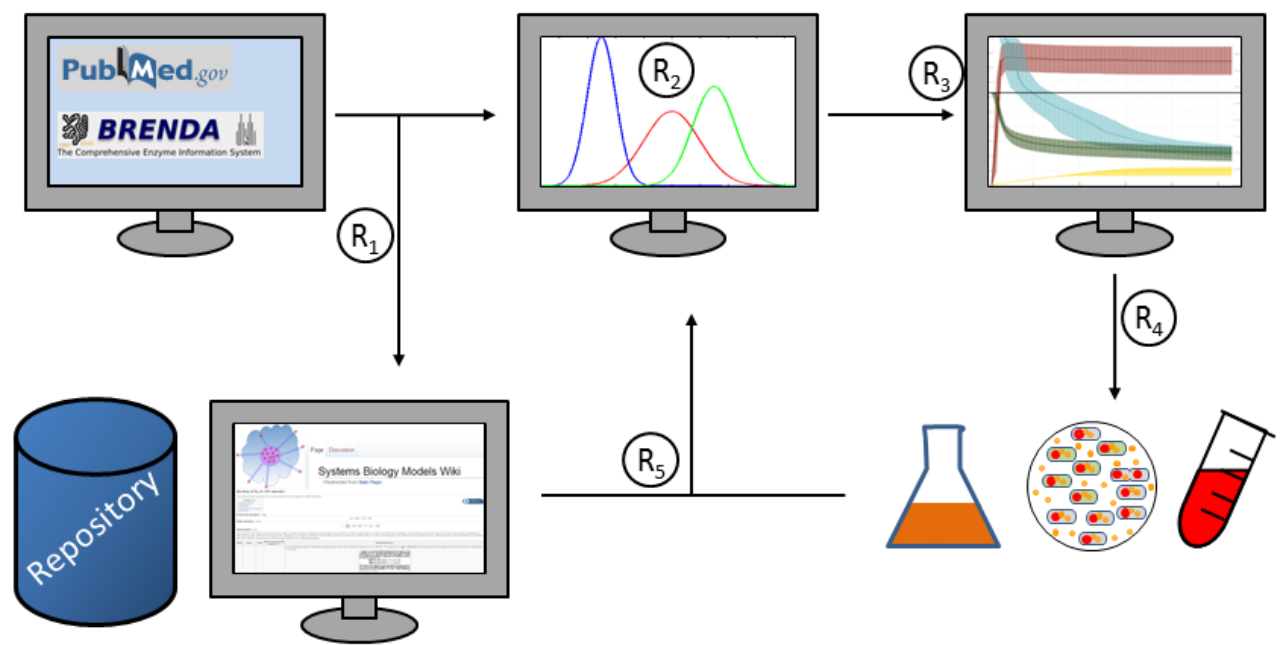

Figure 1: Respectful modelling manifests itself in various steps of the pipeline.

R1: Respect for model construction: full documentation of all modelling decisions and sources in re-usable form for future updating.

R2: Respect for limited knowledge: principled consideration of uncertainty of model parameter data.

R3: Respect for model predictions: quantitative assessment of confidence intervals for all model predictions. 
Many molecular biologists still doubt the relevance of computational models of cellular systems. One sometimes even hears the truism that "All models are wrong, but some are useful” [25], cited as if it meant "all models are false and most are useless". In fact, models are meant to be explicit descriptions of our implicit knowledge about the function of a biological system. Therefore, they are ideally no more wrong than less-formal reasoning about the same system, yet they offer numerous advantages: most importantly, they put any assumptions about how a cellular mechanism works out into the open for everyone else to check and criticize, and they allow predictions about non-obvious (“emergent”) behaviours of a system that follow compellingly from these assumptions, even when the complexity and non-linearity of the systems make simple back-of-the-envelope arguments impossible.

Yet, beyond the anecdotal observation that models are sometimes flippantly dismissed as "always wrong”, there are more serious indications that computational models of cellular pathways and circuits are not yet fully respected as scientific tools in molecular biology. For example, complex systems models are very rarely updated and developed further; with a few rare exceptions, such as some constraint-based models [26, 27], there are not many examples of "versioned" and iteratively improved models. Moreover, predictions of computational models in systems biology are usually reported without error bars. In contrast, the incorporation of uncertainty in models has long been common practice in other fields [28, 29], from natural hazard insurance to economic forecasts, where the predictions and their associated confidence make a real difference to people's lives. For example, in the prediction of human-induced climate change, error bars and the exploration of multiple scenarios are the dominant feature in all predictions, to the extent that they are even presented and discussed in the popular press [30].

\section{A respectful approach to biological modelling}

So, what is needed to establish the same "respectful" attitude to modelling in biology, so that it can meaningfully contribute to areas such as synthetic biology or personalized medicine? Two aspects seem to be central:

1. Respect for the model itself as a resource that can grow and develop. If we respect our models, we should not treat them as one-off exercises. Instead, we need to make them understandable and reproducible by other scientists and update them regularly and 
continuously rather than discard them once new discoveries are made (or a new PhD student takes on the project). Therefore, reaction mechanisms and parameter values (such as assumed rate constants, enzyme concentrations, or substrate affinities) need to be documented, so that others can build on our work, and models that capture the full breadth of alternative hypotheses need to be maintained, instead of fixating on one "maximum likelihood" or preferred model. In this way, the model can improve iteratively as new data become available, instead of having to be rebuilt each time.

2. Respect for the output of the model and for the predictions it generates. We should strive for accurate predictions that can be used for rational decision-making. When respecting our models, in the sense that we really care about their predictions, we need to care about the associated level of uncertainty, and we need to identify the different levels of confidence associated with each alternative prediction. Otherwise, we could not place reasonable bets on different possible outcomes. Therefore, we need model predictions with confidence intervals, correctly reflecting our current (limited) knowledge about the topology and dynamics of the biological system. We can only ignore confidence intervals as long as we are not interested in the real-world implications of our quantitative model predictions, i.e., when we only treat them as little more than random numbers that illustrate the elegant model-building and analysis algorithms. Once we have a true stake in generating accurate quantitative predictions, because we want to apply them in the design of actual biological experiments or the engineering of real living systems, we will listen much more carefully to what the models have to tell us. Looking for the uncertainty in the model predictions is not a sign of disrespectful mistrust, but an immediate consequence of taking the model serious as a predictive, rather than illustrative, tool.

In fact, these two aspects are closely related. They both imply that our models should not be rigidly fixed but need to capture alternative scenarios, alternative parameter values [31], alternative circuit topologies [32], and generally alternative hypotheses about the biological system that is being studied. A recent study on the energy metabolism of the protozoan parasite Trypanosoma brucei [33, 34], the causative agent of sleeping sickness, illustrates how an explicit treatment of uncertainty can yield new insights even for well-studied model organisms (Box 1). The "respectful modelling" data identify a number of previously unexplored key experiments. In the trypanosome example, for instance, the exact level of permeability of the glycosomes for specific glycolytic intermediates could be determined by a more targeted measurement, or the sensitivity of trypanosomes to the inhibition of glycosomal enzymes with unexpectedly high control coefficients (i.e. triose phosphate isomerase) could be directly measured. 


\section{Box 1: "Respectful modelling” on trypanosome metabolism}

In trypanosome parasites in the human bloodstream, energy metabolism is mostly restricted to glycolysis, which takes place in unique, specialized organelles, the glycosomes; its enzymes are considered promising targets for newly developed drugs against sleeping sickness.

The steps followed during the model design and analysis are depicted in Figure I. Starting from a highly curated model of trypanosomal energy metabolism, the experimental uncertainty of every enzyme kinetic parameter was determined by an extensive exploration of the original literature. Parameter sources, evidence for alternative model topologies (extra reactions), as well as any calculations (e.g., for the parameter means and standard deviations) were documented in a dedicated Wikibased database (A). Several versions of the model were created, in which alternative groups of metabolites with different molecular weight could freely diffuse across the membrane, representing the uncertainty about possible glycosome permeability resulting from recent evidence that the organelle membrane contains non-selective pores (B).

For each of the alternative models, ranging from very tight to very leaky glycosomes, plausible combinations of parameter values were sampled according to the documented uncertainty by using a random number generator in accordance with each parameter's assumed probability distribution. This resulted in a large collection of model variants, each using a different set of parameter values (C). The ensembles of models were then subjected to the same types of analysis as traditional dynamic models, e.g. determining steady-state concentrations of metabolites and calculating the control coefficients of enzymatic reactions that could identify the most promising drug targets (D).

The analysis of the resulting ensembles of model predictions provided several interesting insights that had remained "hidden" in classical maximum-likelihood analyses of individual models. For example, it revealed unexpected fragilities in the existing models. Two metabolites, 3-phosphoglycerate and pyruvate, seemed to accumulate to impossible concentrations in many of the models, indicating that critical regulatory loops are probably still unaccounted for in our current understanding of trypanosome metabolism. Moreover, control of glycolytic flux seemed to be more widely distributed between several key reaction steps, rather than being largely restricted to the ratelimiting glucose uptake transporter. The results

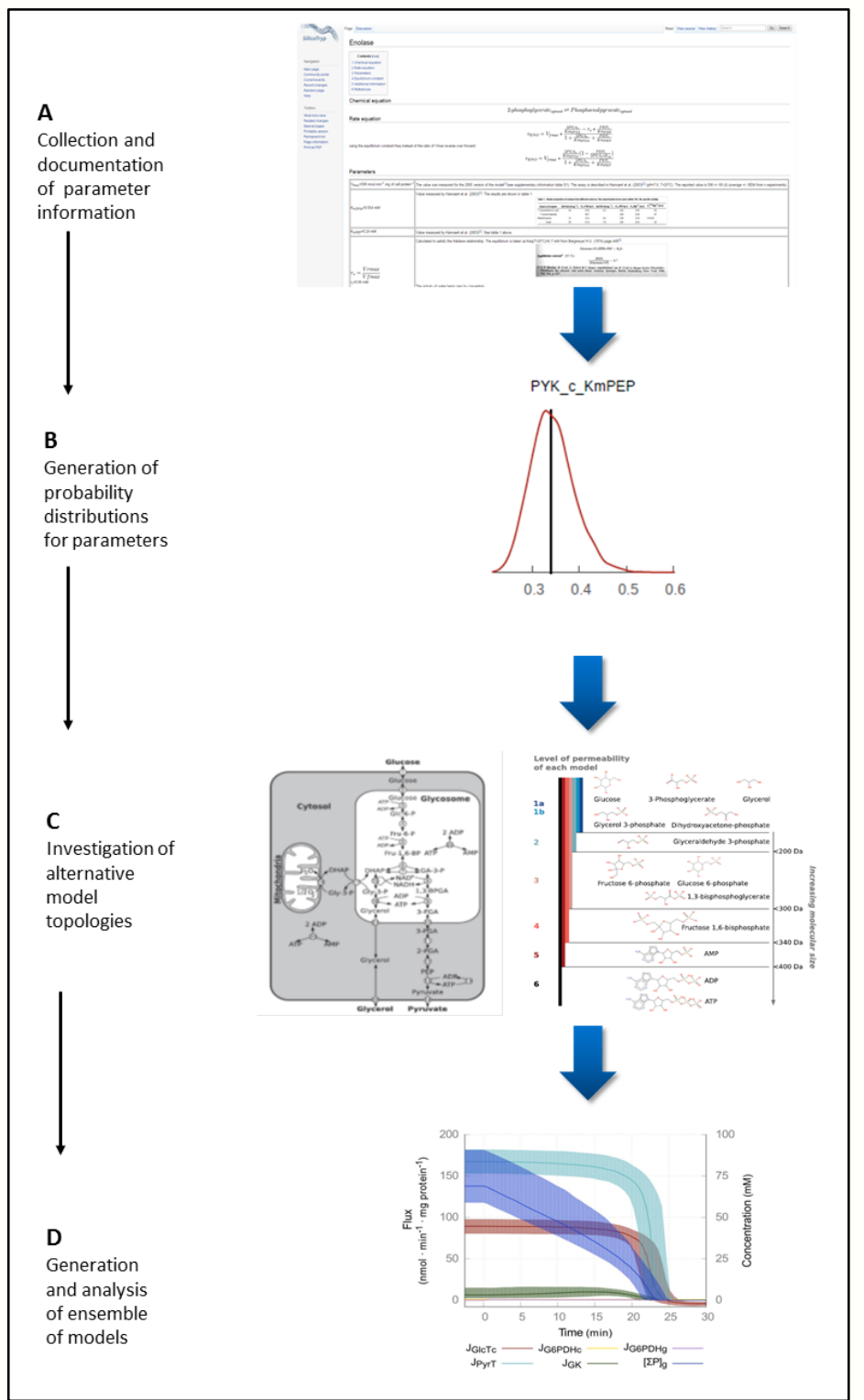

Figure I: Flow chart of steps followed during the dynamic modelling of $T$. brucei energy metabolism with explicit consideration of parameter uncertainty. [33, 34, 50] also showed that the models that predicted steady-state metabolite concentrations and fluxes that most closely matched experimental observations, for the largest number of plausible parameter sets, were the ones with glycosomes permeable to small metabolites up to the size of fructose 6-phosphate and fructose 1,6-bisphosphate. This result challenges the current consensus view of trypanosome glycolysis that critically depends on highly controlled trans-membrane fluxes but is in good agreement with the presence of recently discovered unspecific glycosomal pores. 
Therefore, these two aspects represent different levels of alternatives: on one hand, the degree of confidence in our predictions can be determined by examining ensembles of equally plausible models. On the other hand, models can be updated and re-used as our biological understanding evolves, by changing our assumptions about which models (and parameter values) are plausible and which are not.

The advantages of respectful modelling are obvious:

1. It quantitatively indicates each prediction's robustness, which is helpful for identifying the most suitable experiments to carry out next: do we already have enough confidence in a prediction to build an expensive study around it, or are there specific uncertainties that we first need to reduce by targeted measurements?

2. It enables collaborative and iterative work on model building and model improvement by explicitly identifying and documenting alternative model structures and parameter values.

3. It allows managing alternative hypotheses about the functions of complex biological systems within a unified modelling framework, instead of having slightly different, incompatible, and often-incomparable models associated with each hypothesis.

4. Finally, and perhaps most importantly, it makes modelling approaches available for systems in which quantitative information is incomplete and uncertain, thus unveiling otherwise inaccessible biological phenomena. This may also help bridging the conceptual gap between quantitative and qualitative modelling [35].

\section{Respectful modelling showcases and applications}

Several important steps have recently been taken to provide the ingredients for a respectful modelling approach.

1. Model descriptions are being standardised. This practice is becoming applied to every aspect of the model building process, from file formats to variable names and equation structures and to mandatory archiving of the resulting models. The Systems Biology Markup Language (SBML) [36], an XML-based machine readable language, is supported by almost all software tools in the field and is prevailing as a model representation format that facilitates model sharing and replication. Additionally, models are starting to comply with some basic community guidelines that describe the minimum information that needs to accompany a published model (MIRIAM) [37] along with conventions that facilitate data 
integration in model building, such as SBtab [38]. Moreover, centralised model repositories such as BioModels [39], JWS Online [40], CellML Model repository [41] and the BiGG database [42] have been created to enable the distribution and curation of biological system models.

2. Parameter information is being rigorously documented. The inclusion of supplementary material on the sources and values of the parameters (e.g., enzyme and substrate affinities, transcription rates, etc.) in published models is increasing [43-46]. For example, recently published updated models of central carbon metabolism in trypanosome parasites included a Wiki page dedicated to each biochemical reaction of the system [33, 34, 47]. In this way, detailed information on the sources for each parameter value was provided, along with descriptions of the underlying calculations and assumptions, as well as alternative model versions with different topologies. This approach not only increases the accountability of modellers for the critical decisions made during model building; it also greatly facilitates the reconstruction, validation and updating of models by successive generations of researchers.

3. Moreover, the detailed documentation of parameter sources enables the quantification of the uncertainty associated with each value, so that model predictions can take these uncertainties into consideration intuitively. A formal acknowledgement of uncertainty in computational models of biological systems not only describes the modellers' beliefs and confidence in model structure and model parameters, but it also explicitly identifies alternative structures and parameter values and their associated plausibility [33, 34]. This approach mirrors the process of scientific progress by continuously exploring alternative hypotheses. Thus, rather than paralyzing the model's analysis, the explicit acknowledgement of uncertainty actually enables the flexible evolution of biological models, which at each stage honestly represent our current knowledge about a biological system. This is in contrast to traditional modelling approaches, where alternative hypotheses and assessments of uncertainty are managed only in an ad hoc process taking place implicitly in the brains of the modellers and their collaborating expert biologists. Furthermore, acknowledging uncertainty and integrating it during the model building phase allows making predictions that are associated with specified confidence intervals, which can guide further experimentation [48, 49].

4. To transition from the collected information about parameter uncertainty to the resulting quantitative assessment of our confidence in specific model predictions, data-driven parameter sampling strategies have been formulated [33, 34, 47, 50, 51]. They employ informative distributions to describe what the modellers (and their biologist collaborators) 
consider as plausible values for each parameter. These distributions correspond to the priors

in a Bayesian statistical framework [52, 53], and can indeed be used for a Bayesian statistical analysis $[54,55]$ to update the parameter values when new experimental evidence becomes available, but they most importantly can be used in the next step to create an entire ensemble of plausible models by sampling values for each parameter from its corresponding distribution (Box 2). Experimental data, biological background knowledge, and biophysical plausibility (Box 3) can all contribute to defining the most appropriate distributions that capture our current state of knowledge accurately, not exaggerating the uncertainty, but also not being overconfident about specific values or connections in the network.

\section{Box 2: Generating probability distributions to describe uncertainty}

Describing the uncertainty associated with our knowledge of parameter values in molecular systems models is challenging.

The natural choice for the shape describing the range of plausible parameter values is often a log-normal distribution: there will be a most likely value (the mode of the distribution), negative values are not allowed, and the distribution is symmetrical, in the sense that values that are $x$ times larger than the most likely estimate, are just as plausible as values that are $x$ times smaller (Fig. I). More specifically, the mode is the value $\mathrm{x}_{\mathrm{o}}$ for which the condition $\mathrm{f}\left(\mathrm{x}_{\mathrm{o}} * \delta\right)=\mathrm{f}\left(\mathrm{x}_{\mathrm{o}} / \delta\right)$ is fulfilled for all real numbers $\delta$ (where $\mathrm{f}$ is the probability density function).

In contrast to common assumptions, the log-normal distribution is ubiquitous in nature across different fields and often a far better description of the data than a normal distribution. This is particularly seen in the medical and biological sciences, when the mean values are low and the variances are large, yet the values cannot be negative, whether they be latency periods of infectious diseases in epidemiology, species abundances in ecological studies, or enzyme kinetic parameters as in the

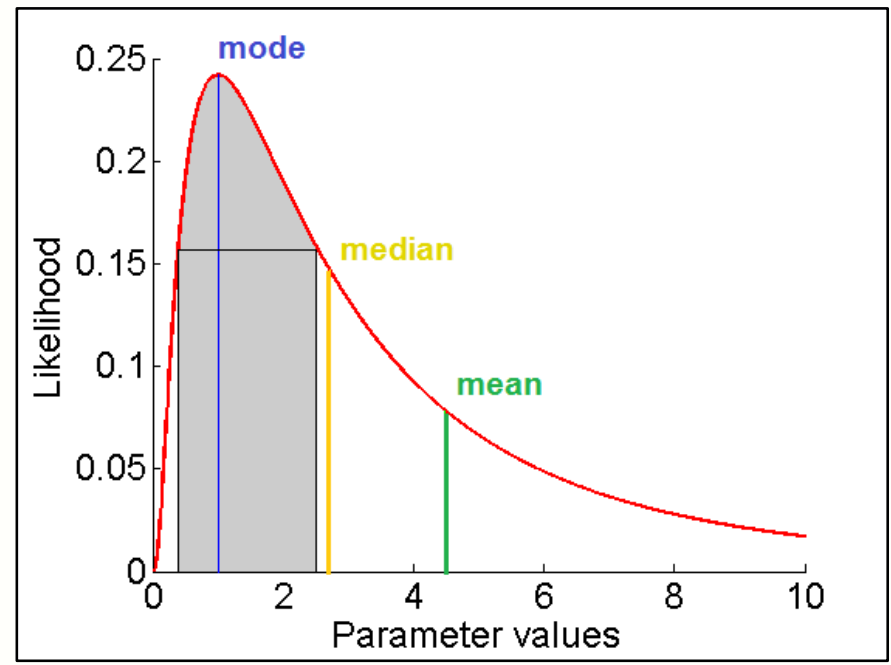

Figure I: The properties of a log-normal distribution with $\mu=\sigma=1$. The mode represented by the blue line is equal to 1 , the mean is equal to 4.48 (green line), and the median is equal to 2.7 (yellow line). For any value of $\delta$ the product and the quotient of the mode and $\delta$, have equal probability of being sampled $(f(1 * \delta)=f(1 / \delta)$; illustrated for $\delta=2.5$ by the edges of the grey area). log-normal distribution

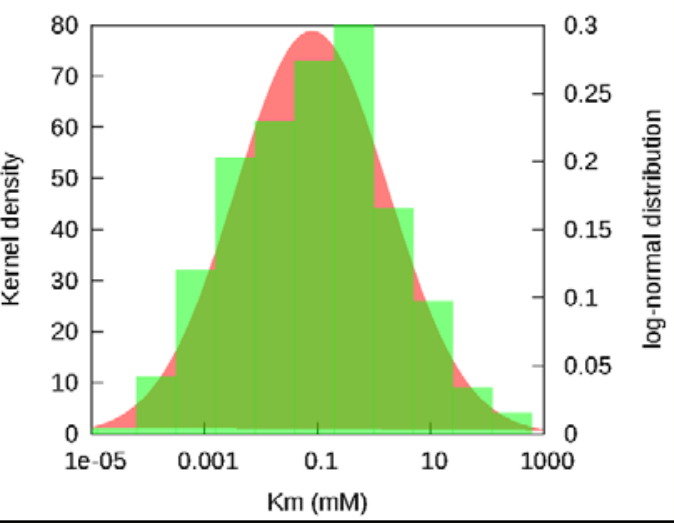

Figure II: Distribution of the $K_{m}$ values retrieved from the BRENDA database. $K_{m}$ values retrieved from the database are shown in green and the corresponding fitted log-normal distribution is shown in red. [34] Kernel density of $\mathrm{Km}$ values retrieved by BRENDA

present discussion [56]. The reason for this predominance of log-normal distributions is simple: when the change in a variable (observable) at each step of the process is proportional to its current value (i.e., when the process follows Gibrat's law of proportionate effect), the resulting observations will be log-normally distributed [57]. In the life sciences, biochemistry, biophysics and population ecology are disciplines where processes characterized by Gibrat's law are most obvious: reaction velocities, surface and volume measurements, and population growth (also at the molecular level) are regulated by factors that act in a multiplicative way, rather than in the additive way that would be required for data to become normally distributed.

The challenge for the biological systems modeller is to decide on the appropriate values describing the distribution: what is the most likely value (described by the mode of the distribution) and how rapidly does the plausibility of the values decrease when moving away from this value (described by the standard deviation). These in turn determine the location and scale parameters $\boldsymbol{\mu}$ and $\boldsymbol{\sigma}$ of the log-normal distribution. In many cases, a good estimate of the most likely parameter value exists, e.g. from actual experimental measurements. In other cases, related parameters have been measured, e.g., the kinetics of similar enzymes or even of the same enzyme in different species or conditions. In the extreme case, no measurements are available at all; but even in that 
case, an informed guess is usually possible. For instance, the most plausible $\mathrm{K}_{\mathrm{m}}$ value of an uncharacterized novel enzyme might be the average of all $\mathrm{K}_{\mathrm{m}}$ values ever recorded in a comprehensive enzyme database like BRENDA, which comprehensively records available experimental data on enzyme kinetic parameters from all domains of life. The spread of the distribution, its standard deviation, would in such a case reasonably be determined by the range of all reported values (Fig. II).

It is also necessary to decide if there are any hard (biophysical) thresholds, beyond which a parameter value is not only implausible, but impossible; in other words, if the distribution should be truncated at the extremes. In every case, it is important to remember that determining plausible parameter distributions is not a mechanical exercise but has to be based on actual biological and biophysical reasoning. If there are arguments to support the idea that a given log-normal distribution does not match the expected range of plausible values, it needs to be adjusted accordingly.

\section{Box 3: Thermodynamic consistency}

Once the plausible values for each parameter have been described, there is another important factor that needs to be considered to decide if combinations of parameters are plausible: thermodynamic consistency. For example, imagine a reaction that is known to have an equilibrium constant very close to 1 ; i.e., its standard Gibbs free energy $\Delta \mathrm{G}^{0}=0$. We are trying to determine the kinetic parameters for the forward and backward component of this reaction, and we don't know much about the rate of the reaction, so we sample each of the two parameters from a very broad distribution. If we do not take the additional thermodynamic information into account, we will often end up with sampling the forward reaction rate from the "fast" end of the spectrum, and the backward rate from the "slow" end, or vice versa. Thermodynamic consistency requires that we discard such samples and only keep samples where the two reaction rates are very similar (how similar will in turn depend on our uncertainty about the equilibrium constant) (Fig. I).
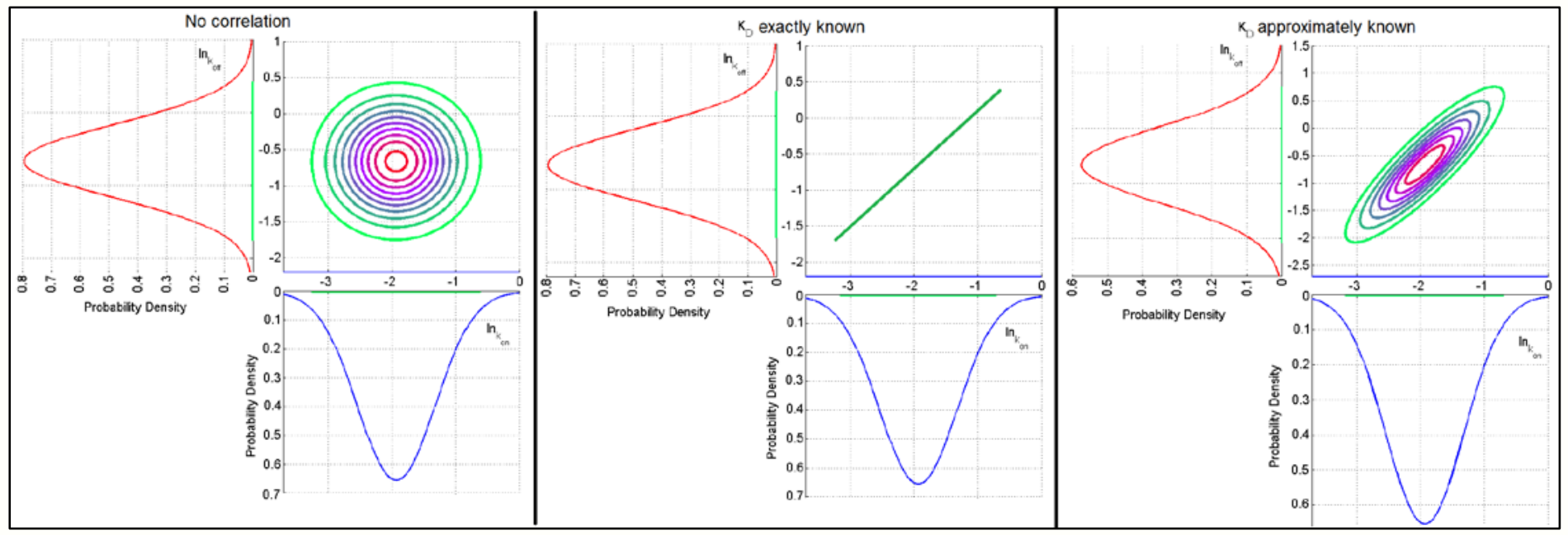

Figure I: Sampling strategy for maintaining thermodynamic consistency of parameter sets. In order to ensure the thermodynamic feasibility of a parameter combination in the case of interconnected parameters (i.e. forward and backward reaction, $k_{\text {on, }} k_{\text {off) }}$ a bivariate distribution is created. When the two marginal distributions are non-correlated, the generated points that represent parameter pairs form a circle. When the equilibrium dissociation constant, $K_{D}$, is exactly known, the parameters $k_{\text {on }}$ and $k_{\text {off }}$ are tightly correlated through the $K_{D}$ value, and the points form a straight line [51]. Finally, if $K_{D}$ is approximately known (a distribution of values for $K_{D}$ exists) the resulting points of the bivariate system form an ellipse. The thickness and orientation of the ellipse depend on the magnitude of the correlation between the two marginal distributions and on the degree of uncertainty on the values of $k_{\text {on, }} k_{\text {off }}$ and $k_{D}$ [Tsigkinopoulou et al., unpublished data]. This case represents the realistic scenario when modelling, as usually the parameter values are approximately known. The first case (no correlation) does not respect thermodynamic consistency and is therefore undesirable. The second case, although taking into account the dependency of the two parameters, is in most cases unrealistic since the value of a parameter such as the equilibrium constant is rarely exactly known.

5. Approaches that explicitly acknowledge model uncertainty have been developed and employed [31], such as Markov Chain Monte Carlo (MCMC) methods [58-60], ensemble 
modelling [61, 62], and global sensitivity analysis [52, 63, 64]. Such techniques are based on the concept of sampling parameters from their associated probability distributions and thus creating a collection of models can undergo further analysis in a similar way as typical dynamic models. In contrast to analyses of the local effects of variable parameter values around their preferred value, the entire resulting landscape of solutions can be surveyed without focusing only on one optimal solution, which may in the future be rejected once new data become available. This approach is also highly preferable over methods that try to fit a single "maximum likelihood" set of parameters based on the best match to experimental data [54]. Such a "fitting” strategy is very popular in applications including transcriptional dynamics [65, 66], epigenetics [67], neuronal dynamics [68], and population-level epidemiology [69]. However, fitted parameters are also very likely to be trapped in transient and spurious global optima, given that the likelihood surface for complex biological systems is inherently extremely rugged (convex), and are always in danger of leading to overfitting and a failure to identify alternative hypothesis that could explain the results equally well[1, 31]. Moreover, parameter values, once fitted, are rarely updated in the face of new experimental results. Avoiding fitting parameter values according to the maximum likelihood is particularly important in the case of biological models: their complexity and non-linearity lead to a highly non-convex (i.e. rugged) likelihood surface, which means that models with considerably different sets of parameter values can have quite similar likelihoods (Fig. 2). If one of them is preferred based on a momentarily higher likelihood, alternative options are easily overlooked later on. In order to avoid this pitfall, all options are kept in view in a respectful modelling approach, so that the model can be easily adapted. In this regard, and in important details of the parameter distributions [70], the respectful modelling approach closely approximates the Bayesian inference processes supposedly implemented in the human neocortex [71]. 


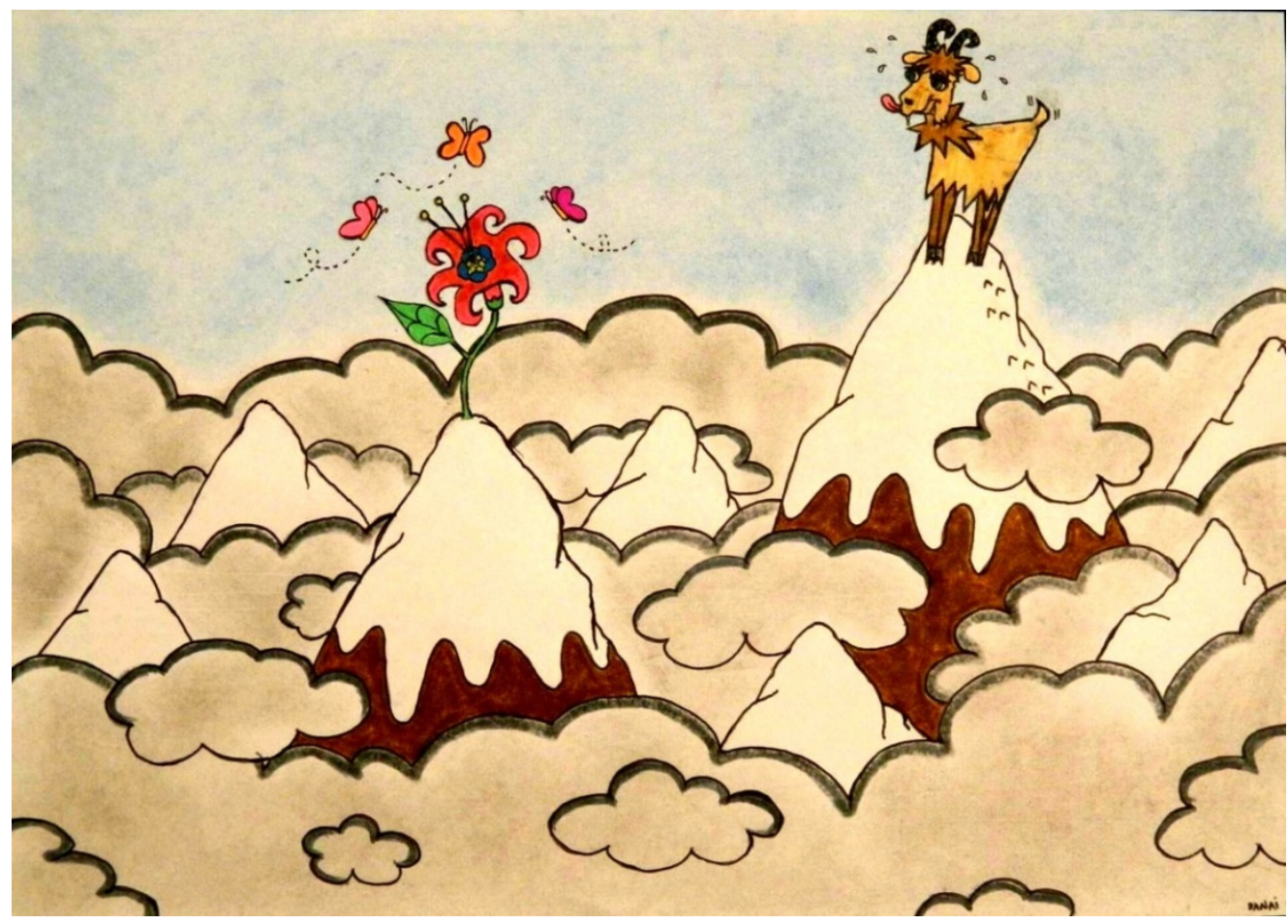

Figure 2: By focusing only on the "best fit solution" a modeller might end up trapped in a local optimum and thus miss interesting alternatives which in the future might be supported by new experimental data. Instead, by surveying the entire landscape of solutions without focusing on one particular peak, respectful (Bayesian) modelling remains adaptable to future developments, especially when studying complex systems with rugged likelihood surfaces, where the availability of new experimental data can result in rapid changes in the relative likelihood of alternative model scenarios. (Courtesy of Danai Triantafyllopoulou)

\section{Conclusion}

It is probably clear by now that all of these developments offer reasonable and useful modifications of the way we build and analyse computational models for molecular biology. But why will they make a difference? They get us closer to the ideal expressed in another famous saying about computational modelling: "Models are not meant to be descriptions, pathetic descriptions, of nature; they are designed to be accurate descriptions of our pathetic thinking about nature” (J. Black, cited in [72]). To achieve this ideal, each model has to allow us to capture the "pathetic" aspects of our thinking, the uncertainties and incompleteness of the evidence, and to evolve as our thinking evolves on the basis of new experiments [73, 74]. In the future of molecular systems biology and modelling, nothing is certain except uncertainty itself (see Outstanding Questions). The increasing use of automated model building strategies will only increase the challenge [75], as models grow in size and the specific refinement of individual parameters by targeted experiments becomes even less feasible than it is now. The adoption of a respectful modelling framework will promote and facilitate collaboration within the biological community, stimulating the use of models as a 
tool for fundamental research, but also as a valuable guide for the predictive engineering of biological systems and their informed manipulation by increasingly personalized drugs.

\section{References}

1 Almquist, J., et al. (2014) Kinetic models in industrial biotechnology - Improving cell factory performance. Metab. Eng. 24, 38-60

2 Wolkenhauer, O. (2014) Why model? Frontiers in Physiology 5

3 Fisher, A.K., et al. (2014) A review of metabolic and enzymatic engineering strategies for designing and optimizing performance of microbial cell factories. Comput. Struct. Biotechnol. J. 11, 91-99

4 Lee, J.W., et al. (2012) Systems metabolic engineering of microorganisms for natural and non-natural chemicals. Nat. Chem. Biol. 8, 536-546

5 Dai, Z. and Nielsen, J. (2015) Advancing metabolic engineering through systems biology of industrial microorganisms. Curr. Opin. Biotechnol. 36, 8-15

6 Chung, B.K.-S., et al. (2013) Genome-scale in silico modeling and analysis for designing synthetic terpenoid-producing microbial cell factories. Chem. Eng. Sci. 103, 100-108

7 Ahn, J.H., et al. (2016) Production of succinic acid by metabolically engineered microorganisms. Curr. Opin. Biotechnol. 42, 54-66

8 Iadevaia, S., et al. (2010) Identification of optimal drug combinations targeting cellular networks: integrating phospho-proteomics and computational network analysis. Cancer Res. 70, 6704-6714

9 Breitling, R. and Takano, E. (2015) Synthetic biology advances for pharmaceutical production. Curr. Opin. Biotechnol. 35, 46-51

10 Takano, E. and Breitling, R. (2015) Synthetic Biology of Antibiotic Production. In Reviews in Cell Biology and Molecular Medicine (RA, M., ed), Wiley-VCH Verlag GmbH \& Co. KGaA

11 Fard, A.T., et al. (2016) Not just a colourful metaphor: modelling the landscape of cellular development using Hopfield networks. NPJ Syst. Biol. Appl. 2, 16001

12 Fey, D., et al. (2015) Signaling pathway models as biomarkers: Patient-specific simulations of JNK activity predict the survival of neuroblastoma patients. Sci. Signal. 8, ra130-ra130

13 Hu, C.Y., et al. (2015) Generating Effective Models and Parameters for RNA Genetic Circuits. ACS Synth. Biol. 4, 914-926

14 Barbolosi, D., et al. (2016) Computational oncology - mathematical modelling of drug regimens for precision medicine. Nat Rev Clin Oncol 13, 242-254

15 Folger, O., et al. (2011) Predicting selective drug targets in cancer through metabolic networks. Mol. Syst. Biol. 7

16 Yadav, V.G., et al. (2012) The future of metabolic engineering and synthetic biology: Towards a systematic practice. Metab. Eng. 14, 233-241 
17 Stanford, N.J., et al. (2015) RobOKoD: microbial strain design for (over)production of target compounds. Front. Cell Dev. Biol. 3, 17

18 Medema, M.H., et al. (2012) Computational tools for the synthetic design of biochemical pathways. Nat Rev Micro 10, 191-202

19 Hyotylainen, T., et al. (2016) Genome-scale study reveals reduced metabolic adaptability in patients with non-alcoholic fatty liver disease. Nat Commun 7

20 Fong, S.S. (2014) Computational approaches to metabolic engineering utilizing systems biology and synthetic biology. Comput. Struct. Biotechnol. J. 11, 28-34

21 Motamedian, E., et al. (2016) Reconstruction of a charge balanced genome-scale metabolic model to study the energy-uncoupled growth of Zymomonas mobilis ZM1. Mol. BioSyst. 12, 12411249

22 Link, H., et al. (2014) Advancing metabolic models with kinetic information. Curr. Opin. Biotechnol. 29, 8-14

23 Chakrabarti, A., et al. (2013) Towards kinetic modeling of genome-scale metabolic networks without sacrificing stoichiometric, thermodynamic and physiological constraints. Biotechnol. J. 8, 1043-1057

24 Smallbone, K. and Mendes, P. (2013) Large-scale metabolic models: From reconstruction to differential equations. Ind. Biotechnol. 9, 179-184

25 Box, G.E.P. (1979) Robustness in the Strategy of Scientific Model Building. MRC technical summary report: Mathematics Research Center University of Wisconsin-Madison. Defense Technical Information Center

26 Reed, J.L., et al. (2003) An expanded genome-scale model of Escherichia coli K-12 (iJR904 GSM/GPR). Genome Biology 4, R54-R54

27 Swainston, N., et al. (2016) Recon 2.2: from reconstruction to model of human metabolism. Metabolomics 12, 109

28 Peterson, J.T. and Freeman, M.C. (2016) Integrating modeling, monitoring, and management to reduce critical uncertainties in water resource decision making. J. Environ. Manage.

29 Arnone, E., et al. (2014) Parameter Uncertainty in Shallow Rainfall-triggered Landslide Modeling at Basin Scale: A Probabilistic Approach. Proc. Earth. Planet. Sci. 9, 101-111

30 Antilla, L. (2005) Climate of scepticism: US newspaper coverage of the science of climate change. Global Environ. Change 15, 338-352

31 Vanlier, J., et al. (2013) Parameter uncertainty in biochemical models described by ordinary differential equations. Math. Biosci. 246, 305-314

32 Babtie, A.C., et al. (2014) Topological sensitivity analysis for systems biology. Proc. Natl. Acad. Sci. U.S.A. 111, 18507-18512

33 Achcar, F., et al. (2013) Explicit consideration of topological and parameter uncertainty gives new insights into a well-established model of glycolysis. FEBS J. 280, 4640-4651

34 Achcar, F., et al. (2012) Dynamic Modelling under Uncertainty: The Case of Trypanosoma brucei Energy Metabolism. PLoS Comput Biol 8, e1002352 
35 Samaga, R. and Klamt, S. (2013) Modeling approaches for qualitative and semi-quantitative analysis of cellular signaling networks. Cell Communication and Signaling 11, 43

36 Hucka, M., et al. (2015) The Systems Biology Markup Language (SBML): Language Specification for Level 3 Version 1 Core. Journal of Integrative Bioinformatics 12, 266

37 Novere, N.L., et al. (2005) Minimum information requested in the annotation of biochemical models (MIRIAM). Nat Biotech 23, 1509-1515

38 Lubitz, T., et al. (2016) SBtab: a flexible table format for data exchange in systems biology. Bioinformatics 32, 2559-2561

$39 \mathrm{Li}$, C., et al. (2010) BioModels.net Web Services, a free and integrated toolkit for computational modelling software. Briefings in Bioinformatics 11, 270-277

40 van Gend, C., et al. (2007) Data and model integration using JWS online. In Silico Biology 7, 27-35

41 Cuellar, A., et al. (2015) The CellML 1.1 Specification. Journal of Integrative Bioinformatics 12, 259

42 King, Z.A., et al. (2016) BiGG Models: A platform for integrating, standardizing and sharing genome-scale models. Nucleic Acids Res. 44, D515-D522

43 Pai, A. and You, L. (2009) Optimal tuning of bacterial sensing potential. Mol. Syst. Biol. 5, 286-286

44 Mattioni, M. and Le Novère, N. (2013) Integration of Biochemical and Electrical SignalingMultiscale Model of the Medium Spiny Neuron of the Striatum. PLoS ONE 8, e66811

45 Singh, V.K. and Ghosh, I. (2006) Kinetic modeling of tricarboxylic acid cycle and glyoxylate bypass in Mycobacterium tuberculosis, and its application to assessment of drug targets. Theor. Biol. Med. Model. 3, 27-27

46 Smallbone, K. and Corfe, B.M. (2014) A mathematical model of the colon crypt capturing compositional dynamic interactions between cell types. Int. J. Exp. Pathol. 95, 1-7

47 Breitling, R., et al. (2013) Modeling Challenges in the Synthetic Biology of Secondary Metabolism. ACS Synth. Biol. 2, 373-378

48 Engelhardt, B., et al. (2016) Learning (from) the errors of a systems biology model. Sci. Rep. 6, 20772

49 Ruess, J., et al. (2013) Designing experiments to understand the variability in biochemical reaction networks. J. R. Soc. Interface 10

50 Kerkhoven, E.J., et al. (2013) Handling Uncertainty in Dynamic Models: The Pentose Phosphate Pathway in Trypanosoma brucei. PLoS Comput Biol 9, e1003371

51 Liebermeister, W. and Klipp, E. (2005) Biochemical networks with uncertain parameters. IEE P. Syst. Biol.

52 Oakley, J.E. and O'Hagan, A. (2004) Probabilistic sensitivity analysis of complex models: a Bayesian approach. J. Roy. Stat. Soc. Ser. B. (Stat. Method.) 66, 751-769

53 Bernardo, J.M. and Smith, A.F.M. (2008) Bayesian Theory. John Wiley \& Sons, Inc. 
54 Calderhead, B., et al. (2013) Bayesian Approaches for Mechanistic Ion Channel Modeling. In In Silico Systems Biology (Schneider, V.M., ed), pp. 247-272, Humana Press

55 Xu, T.-R., et al. (2010) Inferring Signaling Pathway Topologies from Multiple Perturbation Measurements of Specific Biochemical Species. Sci. Signal. 3, ra20-ra20

56 Limpert, E., et al. (2001) Log-normal Distributions across the Sciences: Keys and Clues. Bioscience 51, 341-352

57 Grönholm, T. and Annila, A. (2007) Natural distribution. Math. Biosci. 210, 659 - 667

58 Hastings, W.K. (1970) Monte Carlo Sampling Methods Using Markov Chains and Their Applications. Biometrika 57, 97-109

59 Schellenberger, J. and Palsson, B.Ø. (2009) Use of Randomized Sampling for Analysis of Metabolic Networks. J. Biol. Chem. 284, 5457-5461

60 Kramer, A., et al. (2014) mcmc_clib-an advanced MCMC sampling package for ode models. Bioinformatics 30, 2991-2992

61 Tran, L.M., et al. (2008) Ensemble Modeling of Metabolic Networks. Biophys. J. 95, 56065617

62 Jia, G., et al. (2012) Ensemble Kinetic Modeling of Metabolic Networks from Dynamic Metabolic Profiles. Metabolites 2, 891

63 Marino, S., et al. (2008) A Methodology For Performing Global Uncertainty And Sensitivity Analysis In Systems Biology. J. Theor. Biol. 254, 178-196

64 Wainwright, H.M., et al. (2014) Making sense of global sensitivity analyses. Comput. Geosci. 65, 84-94

65 wa Maina, C., et al. (2014) Inference of RNA Polymerase II Transcription Dynamics from Chromatin Immunoprecipitation Time Course Data. PLoS Comput Biol 10, e1003598

66 Honkela, A., et al. (2015) Genome-wide modeling of transcription kinetics reveals patterns of RNA production delays. Proc. Natl. Acad. Sci. U.S.A. 112, 13115-13120

67 Bintu, L., et al. (2016) Dynamics of epigenetic regulation at the single-cell level. Science $351,720-724$

68 Amador, A., et al. (2013) Elemental gesture dynamics are encoded by song premotor cortical neurons. Nature 495, 59-64

69 Eggo, R.M., et al. (2016) Respiratory virus transmission dynamics determine timing of asthma exacerbation peaks: Evidence from a population-level model. Proc. Natl. Acad. Sci. U.S.A. 113, 2194-2199

70 Buzsaki, G. and Mizuseki, K. (2014) The log-dynamic brain: how skewed distributions affect network operations. Nat. Rev. Neurosci. 15, 264-278

71 Probst, D., et al. (2015) Probabilistic inference in discrete spaces can be implemented into networks of LIF neurons. Frontiers in Computational Neuroscience 9

72 Gunawardena, J. (2014) Models in biology: 'accurate descriptions of our pathetic thinking'. BMC Biol. 12

73 Kirk, P.D.W., et al. (2015) Systems biology (un)certainties. Science 350, 386-388 
74 Gustafsson, C. and Vallverdú, J. (2016) The Best Model of a Cat Is Several Cats. Trends Biotechnol. 34, 207-213

75 Sunnåker, M., et al. (2013) Automatic Generation of Predictive Dynamic Models Reveals Nuclear Phosphorylation as the Key Msn2 Control Mechanism. Sci. Signal. 6, ra41-ra41

\section{Caption for key figure (Fig.1)}

Respectful modelling manifests itself in various steps of the pipeline.

R1: Respect for model construction: full documentation of all modelling decisions and sources in re-usable form for future updating.

R2: Respect for limited knowledge: principled consideration of uncertainty of model parameter data.

R3: Respect for model predictions: quantitative assessment of confidence intervals for all model predictions.

R4: Respect for model hypotheses: using models to inform real-world experimental hypothesis testing.

R5: Respect for existing models: rigorous strategies for updating based on new experimental evidence. 